\title{
La Política de Compra Pública como Estímulo a la Innovación y el Emprendimiento
}

\author{
Jon Mikel Zabala-Iturriagagoitia ${ }^{1}$
}

Resumen: En los últimos años se ha produciendo una creciente tendencia hacia la incorporación de instrumentos de articulación de la demanda. Uno de los instrumentos que mayor atención ha recibido es el de la compra pública. En este artículo tratamos de ilustrar cómo el sector público puede comportarse de una manera estratégica, considerando la innovación como uno de los principios fundamentales a la hora de realizar dichas compras. Todo ello queda reflejado por medio de cuatro iniciativas de compras públicas como estímulo a la innovación. Además de ello, ofrecemos un marco conceptual en el que se relaciona la innovación con el emprendimiento y se analiza el potencial de la compra pública para estimular ambos fenómenos.

Palabras claves: compra pública; innovación; emprendimiento.

\section{Public procurement policy as a stimulus for innovation and entrepreneurship}

Abstract: In recent years a major use of demand side instruments to innovation policy has been witnessed, one of this instruments being public procurement. This article aims at providing some evidence on how the public sector can adopt a strategic behavior, considering innovation as one of the main rationales in their public procurement processes. We illustrate this role by analyzing four cases that shed some light on how public procurement for innovation can take place. In addition, we also introduce a conceptual framework relating innovation to entrepreneurship, and the potential role of public procurement as an effective instrument fostering both activities is analyzed.

Keywords: public procurement; innovation; entrepreneurship

Submitted: May $3^{\text {rd }} 2017 /$ Approved: March $28^{\text {th }} 2017$

\section{Introducción}

La compra pública representa una parte muy importante del Producto Interior Bruto (PIB) de un país. De modo ilustrativo, en los últimos años, las administraciones públicas de los países que forman parte de la Unión Europea han invertido entre un 15 y un 20\% de su PIB anual en este tipo de compras (Edquist et al., 2015). A pesar de que los porcentajes varían entre países/regiones, es innegable que la compra pública representa una fuente trascendental de demanda.

La mayor parte de las compras que se llevan a cabo desde las administraciones públicas podría categorizarse como compra pública regular (p.e. adquisición de bienes de consumo estándar). Es decir, las compras públicas carecen, de modo general, de impacto sobre las actividades innovadoras (Edquist et al., 2015). Sin embargo, la inclusión del criterio de la innovación en las compras públicas puede ofrecer mejores resultados para el comprador (Edquist y Zabala-Iturriagagoitia, 2012).

En este artículo consideramos que la compra pública como estímulo a la innovación (CPI) puede constituir uno de los instrumentos más relevantes en el contexto de los grandes retos del siglo XXI. La naturaleza de por sí compleja de dichos retos implica la necesidad de definir políticas e instrumentos que resuelvan algunos de los problemas derivados de los mismos.
Recientemente, las políticas de compras públicas han aparecido con mayor frecuencia y fuerza en las agendas tanto nacionales como regionales (OECD, 2011). Sin embargo, aún no se ha llegado a un escenario en el que se conciban este tipo de instrumentos como potenciales fomentadores no sólo de la innovación, sino también del emprendimiento. Este es precisamente el objetivo de este artículo, a saber, identificar a la compra pública como un instrumento de política pública que puede ofrecer oportunidades que deriven no solo en la generación de innovaciones, sino también en la generación de actividades emprendedoras.

El artículo queda estructurado de la siguiente manera. La sección segunda detalla el papel que desempeña la política de articulación de la demanda. La sección tercera, introduce los diferentes tipos de compra pública en relación con la innovación. La sección cuarta describe la metodología seguida, y detalla las experiencias desarrolladas en diversos países, y que ayudan a ilustrar las anteriores categorías de CPI. La sección quinta dota de un marco analítico que permita identificar las oportunidades que representa la compra pública como un instrumento estimulador del emprendimiento, un campo que no ha sido explorado hasta la actualidad. Finalmente, la sección sexta concluye el artículo ofreciendo las conclusiones derivadas del mismo.

(1) Deusto Business School, Deusto University, Camino de Mundaiz 50, 20012 Donostia-San Sebastian, Spain.

E-mail: jmzabala@deusto.es 


\section{La articulación de la demanda en los sistemas de innovación}

Uno de los principales puntos de partida de los sistemas de innovación radica en la percepción de la innovación como un proceso interactivo, por el cual, las organizaciones - públicas o privadas, desarrollan sus procesos en colaboración con otros agentes o actores del sistema (Edquist, 1997). El concepto de sistema de innovación enfatiza la importancia que tienen los factores relacionados con la demanda, entre los que se incluyen los usuarios finales y la interacciones entre usuarios y productores (Gregersen, 1992). Sin embargo, dicho enfoque no sólo se centra en el papel de la demanda, sino que también se pronuncia respecto a otros determinantes que influyen en el desarrollo y difusión de las innovaciones (Edquist, 2005). En este artículo nos centraremos en el papel que desempeñan las organizaciones con un carácter público, dejando de lado la influencia de otro tipo de agentes privados.

La justificación de la necesidad de intervención pública en políticas de articulación de la demanda queda explicada por los fallos sistémicos y de mercado que están presentes en la mutua relación de dependencia entre la demanda y la innovación (Dalpé et al., 1992; Edler y Georghiou, 2007; Edler, 2009; Gustafsson y Autio, 2011). Estos fallos se deben a que (i) los primeros usuarios tienen unos mayores costes de entrada, (ii) el mercado no reconoce el potencial de los productos y servicios innovadores, y por tanto no constituye una demanda suficiente para su introducción, (iii) los usuarios se encuentran inmersos en el uso de tecnologías anteriores, por lo que el cambio a nuevas trayectorias tecnológicas requiere de fuertes incentivos, (iv) durante los primeros estadios en la introducción de las innovaciones apenas se observan economías de red debido a la poca cantidad de usuarios, (v) los usuarios no son capaces de enumerar sus necesidades, y (vi) existe una incapacidad para traducir los requerimientos de la demanda en términos de nuevos productos y servicios.

Uno de los principales instrumentos de articulación de la demanda es la compra pública. Rothwell y Zegveld (1981) fueron de los primeros autores en estudiar el impacto que tenía la compra pública en comparación con los programas basados en subsidios públicos para las actividades de Investigación y Desarrollo (I+D). Análogamente, Geroski (1990) observó que los instrumentos de compra pública eran más eficientes al estimular las actividades de innovación que los subsidios a la I+D. Más recientemente, Aschhoff y Sofka (2009) han evidenciado que la compra pública de tecnología así como la provisión de una adecuada infraestructura de conocimiento en las universidades tenían un efecto similar sobre las actividades innovadoras. Sin embargo, el impacto de las compras públicas era más notorio en empresas de pequeño tamaño (PYMES) y en aquellas regiones con condiciones económicas desfavorables.

\section{Tipos de compra pública como estímulo a la Innovación (CPI)}

La compra pública regular se produce cuando una organización (pública) adquiere un producto (un bien o servicio, o la combinación de ambos, un sistema) ya existente con anterioridad. Es decir, no se requieren actividades de $\mathrm{I}+\mathrm{D}$ previas, y sólo el precio y la calidad del producto son considerados en la selección del proveedor. Por su parte, se habla de CPI cuando una organización pública abre una invitación para solicitar un producto que no existe en el momento en que se abre la convocatoria, pero el cual podría desarrollarse dentro de un período de tiempo razonable (Edquist et al., 2000). En este tipo de compra pública sí se requiere de la compleción de actividades de $\mathrm{I}+\mathrm{D}$ antes de que la entrega a la entidad o administración demandante pueda ser formalizada.

A continuación presentaremos una clasificación de los tipos de CPI que pueden distinguirse en base a tres dimensiones (Edquist et al., 2000; Edler, 2009; Hommen y Rolfstam, 2009; Uyarra y Flanagan; 2009). La primera hace referencia al usuario final de la CPI:

- La CPI directa se produce cuando el organismo demandante es también el usuario final del producto resultante de la compra. La agencia pública utiliza su propia demanda para inducir a otros agentes del sistema a innovar, en aras de cubrir sus propias necesidades.

- En La CPI catalítica las necesidades se encuentran fuera del ente que actúa como demandante, por lo que la agencia pública adquiere los nuevos productos no para cubrir sus propias necesidades, sino para la satisfacción de aquellas provenientes de otros actores.

La segunda dimensión hace referencia al carácter y grado de innovación demandados:

- La compra pública pre-comercial (PCP) hace referencia a la compra de resultados de investigación esperados, y confina la financiación de actividades de I+D. Sin embargo, no implica la adquisición de ningún producto.

- La CPI adaptativa tiene lugar cuando el producto adquirido es nuevo en el país o región en el que se sitúa la iniciativa. Se requieren de actividades de innovación para adaptar un producto ya existente a las particularidades de la demanda local.

- La CPI evolutiva conlleva el desarrollo de productos totalmente novedosos, por lo que se la relaciona con el desarrollo de innovaciones de carácter radical.

Además de las dos dimensiones anteriores, es preciso añadir una tercera, a saber, el hecho de que la CPI pueda desarrollarse de manera cooperativa. Esta cooperación hace referencia a la correspondencia entre el aprendizaje interactivo y la competición entre posibles proveedores. La CPI cooperativa ocurre cuando el órgano solicitante y los proveedores cooperan durante el proceso de la compra pública (ver Edquist et al., 2000). Esta cooperación público-privada puede tener lugar durante todo el proceso o durante algunas de las etapas del mismo. Por el contrario, la CPI no-cooperativa ocurrirá cuando no exista un diálogo entre las partes implicadas, es decir, en aquellos casos en los que no pueda haber un aprendizaje interactivo entre las organizaciones. En un principio, todas las categorías de CPI son susceptibles de ser cooperativas, por lo que en lugar de tener cinco categorías, se obtendrían un total de diez. 
El hecho de que la cooperación no sea una variable dicotómica sino una cuestión de grado complica la categorización de un tipo de CPI como cooperativo o no-cooperativo. Dado que el aprendizaje interactivo constituye uno de los principios de un sistema de innovación, y por ende, de la mayor parte de las innovaciones, podrá observarse cómo en la mayoría de los casos incluidos en la siguiente sección existe cierto grado de cooperación entre los actores implicados en cada caso, si no durante todo el proceso, durante algunas de las etapas del mismo.

\section{La compra pública como estímulo a la innovación}

La información para la redacción de cada caso ha sido obtenida mediante el acceso a la documentación relevante en cada caso, tales como convocatorias de licitación, literatura científica, documentos de política y evaluaciones y otros materiales escritos e informes a los que se ha tenido acceso. La razón por la que nos hemos centrado en estos cuatro estudios de caso radica por una parte en la experiencia que cada uno de los países (Australia, Noruega, Estados Unidos y Suecia) atesora en la aplicación de la CPI.

Nuestra aproximación debe ser considerada como cualitativa y exploratoria. Dado que uno de los objetivos del artículo es el de ilustrar en qué medida la compra pública puede ofrecer oportunidades que deriven en el fomento de la innovación, el uso de un enfoque cualitativo parece razonable (Knafl y Howard, 1984). Dado el alcance aún limitado de experiencias de CPI, todavía no existe una fuente de datos estadística que permita realizar una evaluación cuantitativa de este instrumento. Es por ello por lo que nuestra información se limita a fuentes de información secundarias.

Son cuatro los casos mediante los cuales se pretende ilustrar el potencial de la CPI. Una versión más detallada del primer caso puede encontrarse en Edquist y Zabala-Iturriagagoitia (2015), mientras que los casos segundo y tercero están descritos en Edquist y Zabala-Iturriagagoitia (2015). La Tabla 1 caracteriza a qué tipo de compra pública se adhiere cada uno de los casos considerados. Como se puede observar, la casilla de CPI catalítica y evolutiva permanece en blanco. Esto es debido a que históricamente la CPI ha sido utilizada principalmente para la satisfacción de las necesidades de las agencias públicas (CPI directa), lo cual refleja que a pesar de que la CPI catalítica es considerada como un instrumento con gran potencial, se encuentra todavía en gran medida en desuso.

Tabla 1. Clasificación de los casos sobre CPI

\begin{tabular}{|c|c|c|c|}
\hline $\begin{array}{l}\text { Carácter del proceso } \\
\text { de compra pública }\end{array}$ & $\begin{array}{c}\text { Compra } \\
\text { pública } \\
\text { Pre-comercial }\end{array}$ & $\begin{array}{l}\text { Compra } \\
\text { pública } \\
\text { adaptativa }\end{array}$ & $\begin{array}{c}\text { Compra } \\
\text { pública } \\
\text { evolutiva }\end{array}$ \\
\hline $\begin{array}{l}\text { Compra pública } \\
\text { directa }\end{array}$ & \multirow{2}{*}{$\begin{array}{l}\text { MVP } \\
\text { (Caso 1) }\end{array}$} & $\begin{array}{l}\text { NødNett } \\
\text { Norge } \\
(\text { Caso 2) }\end{array}$ & $\begin{array}{c}\text { ADS-B } \\
\text { (Caso 3) }\end{array}$ \\
\hline $\begin{array}{l}\text { Compra pública } \\
\text { catalítica }\end{array}$ & & $\begin{array}{l}\text { Hammarby } \\
\text { Sjöstad } \\
\text { (Caso 4) }\end{array}$ & \\
\hline
\end{tabular}

\subsection{MVP}

El Smart SMEs Market Validation Programme (MVP) es uno de los programas incluidos en el plan de innovación del Gobierno de Victoria (Australia), gestionado por el Departamento de Innovación, Industria y Desarrollo Regional (DIIDR). El principal objetivo del MVP es identificar las necesidades tecnológicas de las organizaciones públicas en Victoria y corresponder a las mismas con las capacidades innovadoras de las PYMEs del territorio (Berman y Squire, 2011).

El MVP toma al programa Small Business Innovation Research norteamericano (1982) como su punto de partida, con el que confluye en algunas de sus principales motivaciones. El MVP es considerado por el Gobierno de Victoria como un instrumento de PCP por el cual las PYMEs deben acometer actividades de I+D subsidiadas por la correspondiente agencia pública para poder dotar de soluciones a las mismas. El programa está dividido en tres etapas: (i) especificaciones y requerimientos tecnológicos, (ii) estudio de viabilidad, y (iii) testeo del concepto o solución.

El proceso comienza cuando una agencia pública de Victoria identifica una necesidad tecnológica específica asociada a su misión y para la cual no existe solución comercial disponible. Una vez definidas las especificaciones y requerimientos tecnológicos, se incluyen los mismos en un concurso público por el cual se invita a las PYMEs de la región a que propongan soluciones que puedan cubrir dicha necesidad tras la correspondiente y necesaria etapa de I+D. Durante la segunda etapa, las PYMEs desarrollan sus propias aplicaciones, de forma que aquellas que sean evaluadas positivamente recibirán una financiación de 100.000 AU\$ del DIIDR para el desarrollo de su estudio de viabilidad $\mathrm{y}$ de las actividades de $\mathrm{I}+\mathrm{D}$ asociadas al mismo. Hay que indicar que el MVP ofrece la posibilidad de financiar a más de una PYME para la misma iniciativa. Tras un período de 3 meses, el estudio de viabilidad es evaluado por la agencia correspondiente junto con otra serie de expertos y miembros del DIIDR. Algunos de los criterios de evaluación que se emplean incluyen el alcance de la I+D incluida en la propuesta, la localización de las actividades de $\mathrm{I}+\mathrm{D}$, los recursos requeridos para la misma, sus hitos, el personal, los gastos y la financiación y el futuro plan de comercialización.

En caso de que la propuesta sea evaluada positivamente, la PYME recibe un total de 1,5 millones AU\$ para el desarrollo y posterior testeo de su concepto (2 años). La PYME conservará todos sus derechos de propiedad intelectual, mientras que la agencia financiadora retiene una licencia para la explotación de los mismos. En caso de que la agencia acepte la solución propuesta, las obligaciones de la misma en este caso de PCP se consideran terminadas, sin necesidad de que haya un compromiso para que dicha agencia adquiera la solución obtenida (Berman y Squire, 2011).

La primera ronda de solicitudes fue abierta en 2009. Dentro de la primera etapa se recibieron un total de 74 propuestas con especificaciones y requerimientos tecnológicos provenientes de 27 entidades públicas. A pesar de que la colaboración (entre PYMEs o entre PYMEs y universidades u otros organismos públicos de investigación) no era considerada como uno de los criterios de evaluación, 85 PYMEs (69\% 
de todas las propuestas recibidas) señalaban su intención de cooperar con otra empresa, universidad o centro de investigación en la búsqueda de una solución al problema/necesidad identificado. 19 de estas invitaciones progresaron a la segunda etapa, la cual atrajo un total de 124 propuestas de PYMEs de la región. Tras su correspondiente evaluación, nueve proyectos pasaron a la tercera y definitiva etapa de desarrollo de prototipos y conceptos.

La primera ronda del MVP ha terminado recientemente por lo que no es posible todavía evaluar su impacto económico. Sin embargo, sí se pueden observar ciertas particularidades. Una de ellas es el alto nivel de cooperación entre organizaciones de diverso tipo, así como la alta representatividad de aquellas empresas con menos de 7 empleados (Berman y Squire, 2011, p. 101). Finalmente, resaltar la gran cantidad de propuestas (un total de 70) recibidas desde PYMEs sin un historial previo en la recepción de fondos públicos de I+D. Esta última figura es particularmente significativa, ya que indica que el programa ha servido para apoyar la entrada de nuevos agentes en el sistema de innovación.

\subsection{NødNett Norge}

El proyecto NødNett Norge constituye un caso de CPI cooperativa ya que trataba de dar solución a las necesidades (comunes) de varias organizaciones públicas (p.e. bomberos, brigadas contra incendios, servicios de salud, fuerzas de policía, etc.). El objetivo era establecer una red de radio digital móvil que fuera capaz de coordinar las redes de comunicación análogas e independientes de los cuerpos de seguridad de Noruega. El Ministerio de Justicia y la Policía fueron designados como sus principales solicitantes. Estos agentes cooperaron activamente con otra serie de agencias, como la organización para la defensa logística, la policía del distrito de Sor-Trondelag, las brigadas antincendios de Trondheim, Klaebu, Malvik y Melhus, el hospital de St. Olav y Telenor (Lyngstøl, 2004). Respecto a su grado de innovación, se puede categorizar al proyecto como adaptativo, ya que conllevó la modificación de una tecnología ya existente.

El proceso de compra pública puede dividirse en dos grandes etapas, una primera de carácter pre-comercial y el proceso de CPI en sí mismo. La etapa pre-comercial tuvo lugar entre 1995 y 2004, periodo en el cual se desarrollaron tres tipos de actividades: estudio previo (1995-1996), estudio de viabilidad (1998-2001) y proyecto piloto (2000-2004). Telenor era el responsable de su coordinación, gestionado por la defensa nacional Noruega (Hommen, 2005).

El estudio de viabilidad derivó en la selección del estándar Europeo TETRA (TErrestrial Trunked Radio), el cual ya había sido implementado en varios países del área de Schengen. Durante el desarrollo del proyecto piloto, se implantó una instalación experimental en el área de Trondheim en Julio de 2000, el cual integraba la red TETRA con las redes de comunicación ya disponibles con anterioridad por la policía, los cuerpos antincendios y el departamento de salud. Con ello se daba por concluida la etapa pre-comercial en Junio de 2003.

El proceso de CPI comenzó en Noviembre de 2004 cuando el Gobierno Noruego presentó una estrategia para el establecimiento de una red nacional digital de radio para las brigadas antincendios, la policía y los servicios de salud. El parlamento autorizó al Ministerio de Justicia a abrir el proceso de recepción de propuestas y así determinar el beneficiario de dicha ayuda, responsable a su vez de cubrir las necesidades de comunicación de dichas agencias en 54 municipalidades. El proyecto estaba totalmente financiado por fondos incluidos en los presupuestos generales, de forma que el estado se convertiría en el propietario final de la totalidad del sistema, siendo los usuarios finales pertenecientes a los cuerpos de bomberos, policía, servicios de salud, así como otras organizaciones cuya participación era consideraba necesaria en casos de emergencia, tales como defensa, aduanas, prisiones y algunas ONGs.

Las especificaciones sobre el funcionamiento de la red sólo incluían los requerimientos funcionales de la misma desde el punto de vista del usuario. Sin embargo, éstos fueron detallados a tal extremo que incluían más de 4.000 especificaciones, lo cual dejaba poco margen de maniobra a los potenciales proveedores (Sylvest, 2008).

En Diciembre de 2005 se abrió un concurso público, tras el cual se recibieron cinco propuestas. Tras la evaluación de las mismas, en la cual el precio fue uno de los principales criterios (Sylvest, 2008), Siemens Networks Norway AS fue seleccionado como proveedor en 2006, y el acuerdo entre las partes fue firmado en Marzo de 2007.

El desarrollo del proyecto se llevaría a cabo en dos etapas, en la primera de las cuales se integrarían los sistemas en el este de Noruega, incluyendo Oslo, mientras que en la segunda se cubriría el resto del territorio (Directorate for Emergency Communication, 2007). El proyecto tuvo un impacto directo sobre la generación de nuevos puestos de trabajo en Nødnett Norway, así como mejoras en la eficiencia y en la provisión de servicios de emergencia (Sylvest, 2008, p. 76).

\subsection{ADS-B}

La compra pública en los Estados Unidos tiene lugar a dos niveles, el federal y el estatal. Las reglas que gobiernan las compras públicas de todas las agencias federales están incluidas en la "Federal Acquisition Regulation" (FAR). La FAR y otras regulaciones no son aplicables a las agencias estatales, las cuales tienen un control total sobre sus propios instrumentos de compra pública. Sin embargo, muchas de las agencias federales sustituyen y/o complementan las normas incluidas en la FAR con normas análogas que adaptan los contenidos de la FAR a sus necesidades específicas (p.e. Departamento de Energía, Departamento de Educación, Agencia para la protección del medio ambiente, etc.).

Dos de los principios fundamentales de la FAR, y por lo tanto, considerados en todas las compras realizadas por las agencias federales, son el coste y el apoyo a procesos competitivos. El criterio de la innovación, o la prioridad a procesos innovadores no es por lo tanto promovido por la FAR (Vonortas, 2015). Mientras que la FAR, así como sus adaptaciones federales, proveen normas que estandarizan los procesos de compra pública, las políticas de compra pública son responsabilidad de cada agencia federal, la cual es responsable de definir sus propias especificaciones y de evaluar las propuestas recibidas.

Uno de los casos en los que la innovación fue considerada como uno de los resultados intencionados de la compra pública fue el "Automatic Dependent Surveillance-Broadcast program" (ADS-B) de la Admi- 
nistración de la Aviación Federal (FAA) (Vonortas, 2015). El objetivo del ADS-B consistía en desarrollar una estrategia que se alineara con el sistema NextGen (Next Generation Air Transportation System) y que supusiera un valor añadido para la Agencia Nacional del Espacio Aéreo. El ADS-B permitiría identificar la localización de un avión utilizando sistemas de navegación GPS y emitiendo continuamente la localización precisa de cualquier avión a otros aviones así como a controladores aéreos. Este caso constituye un claro ejemplo de CPI directa en la cual una agencia pretende cumplir sus propias misiones. El proceso de compra pública de la FAA comenzó con la difusión del programa por medio de los denominados 'industry days', los cuales permitieron a las empresas interesadas conocer así como contribuir a identificar y definir los detalles y funciones del sistema. Los detalles de las especificaciones eran funcionales, con la idea de dotar de una mayor flexibilidad a los participantes.

La FAA publicó una solicitud de licitación en Noviembre de 2006, en respuesta a la cual fueron preseleccionadas tres propuestas. La agencia emitió una solicitud de ofertas en Marzo de 2007 para conocer los detalles de las mismas. La evaluación determinó en Agosto de 2007 que la FAA otorgaba el contrato a la propuesta de ITT Corp., por combinar el menor riesgo y posibilitar una implementación menos costosa.

El proceso de implementación del ADS-B se desarrolló en dos etapas: (i) establecimiento de la infraestructura terrestre en asentamientos clave (Golfo de México, Louisville, Philadelphia y Florida del sur); y (ii) desarrollo del equipamiento necesario para desplegar el sistema ADS-B a escala nacional. La primera de las etapas se desarrolló bajo un contrato de costes fijos y un acuerdo por el cual la FAA cubría los gastos derivados de cualquier requerimiento adicional. La segunda etapa fue financiada por un contrato de costes fijos por el cual ITT Corp. debía desplegar suficientes sistemas para poder cumplir con los requerimientos establecidos en la licitación. Dicho contrato también establecía que ITT Corp. debía instalar y mantener el equipamiento terrestre, mientras que la FAA tenía que pagar por los servicios de vigilancia y de transmisión, además de los cargos derivados del equipamiento necesario para la recepción de dicha señal en aviones e instalaciones de control de tráfico aéreo.

Desde su concepción, el ADS-B ha sido considerado por la FAA como una de sus principales tecnologías para la transformación del espacio aéreo norteamericano. La FAA esperaba que el sistema ADS-B conllevaría la sustitución total de los radares terrestres ya que un avión en el que el sistema estuviera disponible proveería tanto a controladores aéreos como a otros pilotos de actualizaciones más rápidas y fiables sobre información aérea, lo cual incrementaría la seguridad de los pilotos. Ello tendría un impacto directo sobre la reducción del espacio aéreo necesario entre aviones, incrementando la eficiencia de dicho espacio además de mejorar la fiabilidad del trabajo de los controladores (McCallie et al., 2011).

\subsection{Distrito de Hammarby Sjöstad en Estocolmo}

En 1998, la ciudad de Estocolmo abrió una concurso medioambiental - Miljötävling 2000, que pretendía reducir el impacto medioambiental en los distritos de Hammarby Sjöstad, Östberga y Skärholmen, y por medio del cual se otorgarían premios para nueva construcción en dichas localizaciones. Con ello, se pretendía reconvertir antiguas áreas industriales en distritos urbanos sostenibles, utilizando un método para la integración y planificación de infraestructuras así como para la implementación de tecnologías innovadoras para el consumo de energía, agua y la gestión de residuos (Lember et al., 2007). Este proyecto constituye el programa de este tipo más amplio y diverso jamás acometido en Suecia tanto en términos de escala como de ambiciones, ya que implicaba la construcción de edificios, infraestructuras y soporte técnico (Kalvet y Lember, 2010, p. 250). Iniciativas como esta contribuyeron a que Estocolmo fuera nombrada capital verde de la UE en 2010.

Durante la construcción del proyecto de Hammarby Sjöstad, el consejo de la ciudad de Estocolmo alentó a promotoras y constructoras a que reforzaran sus principios de reciclaje, consumo y producción de energía. Para el año 2009 ya habían tenido lugar más de 30 proyectos de CPI catalítica, con resultados tanto en términos de innovaciones incrementales como radicales (Bylund, 2006). Los resultados de estos proyectos de CPI son variados. Algunos de ellos fracasaron (p.e. cédulas de gasoil, internet comunitario), otros fueron parcialmente exitosos (medición individual del consumo), mientras que aquellos que derivaron en mejoras incrementales - principalmente en innovaciones de proceso - fueron los más exitosos (p.e. ventanas energéticamente eficientes) (Kalvet y Lember, 2010). Como veremos con posterioridad, las innovaciones radicales tuvieron un mayor impacto en la apertura de nuevos mercados que en la generación de tecnologías radicalmente novedosas (Lember et al., 2011, p. 1384).

El plan de Hammarby incluía la construcción de 15.000 apartamentos y 10.000 oficinas. El programa incluía un amplio abanico de procedimientos de compra pública, desde procesos de negociación divididos en varias etapas, hasta la dotación de varios contratos en paralelo para fomentar la cooperación empresarial. A menudo, también se utilizaron mecanismos de diálogo con proveedores para la elección de la tecnología o procesos de compra más adecuados en cada caso.

Uno de los ejemplos de CPI catalítica es el caso de las ventanas energéticamente eficientes. El grupo de compradores incluía a todas las grandes promotoras y constructoras implicadas en el proyecto de Hammarby Sjöstad. El hecho de tener a dichas promotoras como clientes principales constituía por sí mismo un volumen de producción suficientemente elevado. Las ventanas energéticamente eficientes ya eran producidas con anterioridad en Suecia, pero con un precio superior al deseado en un 30-40\%. Tras recibir propuestas de cuatro grandes fabricantes de ventanas, se aceptó un pedido de la constructora Skellefteå Snickericentral a Överrums Fönsterfabrik (una empresa fabricante de ventanas) en 1999 (Bylund, 2006, p. 92). Las nuevas ventanas fueron adquiridas al mismo precio que las ya existentes en el mercado en aquel momento, pero con las consiguientes mejoras energéticas, lo que implicaba una reducción de la demanda energética en los hogares.

Otro ejemplo lo constituye el desarrollo de nuevas tecnologías para la medición del consumo. El objetivo de dicha iniciativa era la creación 
de un sistema de monitorización de la energía consumida y del coste de distribución en cada apartamento. Ello implicaba la necesidad de desarrollar nuevas tecnologías capaces de medir el consumo eléctrico, de calor, de gas y de agua de modo individual. Dicho proyecto se llevó a cabo entre 1998 y 2000, cuando este tipo de tecnologías todavía no se encontraban disponibles en el mercado Sueco. Las empresas demandantes incluían a 10 constructoras, las cuales definieron los requerimientos técnicos junto con una serie de expertos.

En 1999 se evaluaron las 13 propuestas recibidas, de las cuales cinco fueron invitadas a desarrollar un prototipo. En el año 2000, se efectuaron las correspondientes evaluaciones de los prototipos. Dos de ellos fueron designados como ganadores, y un tercero recibió una mención honorífica. Los prototipos fueron finalmente instalados en 2001 en 500 apartamentos (Bylund, 2006).

\section{La compra pública como estímulo al emprendimiento?}

Esta sección se centra en la relación existente entre la compra pública y el emprendimiento. Para una discusión más detallada de la utilidad de la compra pública como estímulo al emprendimiento, ver Timmermans y Zabala-Iturriagagoitia (2013). A pesar de que no sea posible a día de hoy encontrar contribuciones que dentro de los instrumentos de articulación de la demanda establezcan el nexo entre la compra pública y el emprendimiento, esta sección pretende dar un primer paso en dicha dirección.

\subsection{La relación entre la compra pública y el emprendimiento}

Como se ha evidenciado, las empresas beneficiarias de las iniciativas de CPI fortalecen su capacidad innovadora a través de las actividades de I+D que las encaminan al desarrollo de nuevos productos o sistemas. Por lo tanto, a priori, la CPI debería ser capaz de estimular también el emprendimiento. Sin embargo, la evidencia existente (Edler et al., 2015) muestra cómo las grandes empresas (mayoritariamente) nacionales son las principales beneficiarias de la mayor parte de iniciativas de CPI.

En este artículo definimos el emprendimiento como aquel proceso por el cual se generan nuevas empresas con alto contenido innovador y que abren nuevas oportunidades en varios sectores de actividad. Aquí solo consideraremos como emprendedoras a aquellas empresas que constituyan nuevas entradas en el mercado y que incluyan nuevo conocimiento, el cual derive en innovaciones bien de producto o de proceso. La compra pública puede respaldar aquellas actividades innovadoras que ya están teniendo lugar en el sector, tal y como hemos visto en los cuatro ejemplos de la sección anterior. Sin embargo, también se debe considerar la posibilidad de que la compra pública pueda derivar en el establecimiento de nuevas organizaciones o empresas. En esta sección nos centraremos en este segundo aspecto, considerando así el potencial de la compra pública como estímulo al emprendimiento (CPE).

Una de las razones por las que consideramos que la compra pública puede actuar como CPE es que la misma abre nuevas oportunidades de mercado. Por lo tanto, si como consecuencia de la intervención pública emergen nuevos mercados, sería lógico pensar que de igual modo deberían surgir nuevas formas organizaciona- les. En segundo lugar, la compra pública no solo puede impactar sobre el emprendimiento por la provisión de nuevas oportunidades de mercado, sino también, e indirectamente, transmitiendo oportunidades tecnológicas. Al demandar productos (bienes, servicios o sistemas) innovadores, la agencia pública está indirectamente solicitando a los potenciales proveedores a que desarrollen actividades de I+D que desemboquen en el desarrollo de nuevos productos. En consecuencia, se está estimulando la generación de conocimiento y de tecnología. Por tanto, estas oportunidades tecnológicas pueden simbolizar los incentivos necesarios para la generación de nuevas empresas.

En este sentido, hay que tener en cuenta que los emprendedores, se encuentran a menudo empleados en otras empresas cuando se produce ese efecto de reconocimiento de la oportunidad (Shane, 2003). Es aquí donde debe realizarse un importante inciso, y es que los emprendedores no provienen exclusivamente del sector privado, sino que también proceden del ámbito académico o de organismos públicos de investigación. Por ello, creemos que cuando se anuncian los procesos de compra pública a ciertos agentes - en su mayoría empresas, también deberían incluirse entre dichos potenciales proveedores a universidades y centros (públicos y privados) de investigación, ya que ello no sólo puede derivar en la mejora del conocimiento y de la tecnología requeridas para la satisfacción de la demanda, sino también en un crecimiento de la competencia empresarial por la generación de nuevas organizaciones empresariales tanto en la forma de nuevas spin-off académicas, como de empresas que surgen como consecuencia de un proceso de diversificación de las actividades ya existentes en otras unidades de negocio (intraemprendimiento).

\subsection{Caracterización de los componentes de la compra pública para la estimulación del emprendimiento}

Esta sección pretende abrir un debate sobre la forma en que la compra pública puede estimular el emprendimiento, un campo que no ha sido explorado hasta la actualidad.

\subsubsection{La identificación de las necesidades futuras}

Uno de los principales retos al que se enfrentan los gestores de los programas de compra pública es el reconocimiento de aquellos productos que puedan cubrir las necesidades futuras. En este sentido, la formación y la profesionalización de los gestores y personal implicado en los procesos de compra pública resultan fundamentales (Wintjes, 2012).

Tal y como hemos reflejado, uno de los pilares sobre los que se sustenta la literatura sobre los sistemas de innovación es el aprendizaje interactivo. Como se ha indicado, los procesos de compra pública podrían beneficiarse de la inclusión de una mayor variedad de agentes, no limitando dicho proceso exclusivamente a organizaciones empresariales privadas. Algunos de dichos agentes podrían ser: potenciales usuarios finales, gestores políticos; investigadores y científicos, estudiantes. Desde nuestro punto de vista, los equipos con los que colaboran los gestores de programas de compra pública deberían integrar un adecuado 'mix' de individuos. 


\subsubsection{Requerimientos funcionales vs. técnicos}

El modo en el que la agencia pública formule los requisitos que el nuevo producto deba cumplir tendrá una influencia directa sobre el comportamiento de los potenciales proveedores. Ya hemos indicado cómo la inclusión de una mayor variedad de agentes puede contribuir a una correcta formulación de los requisitos deseados, los cuales consideramos, deberían ser exclusivamente de carácter funcional. Es decir, las características técnicas del producto no deberían ser especificadas por el solicitante, sino que éste debería centrar sus esfuerzos en identificar los requerimientos funcionales que pueden ayudar a satisfacer la necesidad o a resolver el problema social que constituye el motivo de su intervención. Para el solicitante es irrelevante cómo el producto vaya a contribuir a lograr dichos objetivos, por lo que el despliegue de las especificaciones técnicas debe ser dejado en manos del proveedor (Cabral et al., 2006). La experiencia muestra que la definición de un conjunto de especificaciones excesivamente detalladas por el solicitante limita la capacidad y creatividad de los proveedores para proponer soluciones innovadoras (ver sección 4.3. NødNett).

\subsubsection{El papel de la "separación coordinada"}

Uno de los aspectos más relevantes de la CPI y la CPE radica en si la demanda debería agruparse en un único contrato de licitación, de forma que el mercado objetivo sea lo más grande posible - lo cual favorecería a los grandes grupos empresariales, o si por el contrario el mismo debería dividirse en elementos más pequeños, de forma que se dote de estímulos para la participación de organizaciones de reciente creación (emprendedores) o que cuenten con un tamaño menor (PYMEs).
El hecho de crear un único contrato de licitación conlleva que el proveedor sea el responsable de suministrar en exclusiva todos los bienes y servicios relacionados con el mismo, lo que al garantizar una cierta economía de escala, constituye a menudo uno de las principales obstáculos a la participación de PYMEs (European Commission, 2006). Desde nuestro punto de vista este tipo de licitaciones estimulan muy poco la innovación por las siguientes razones: (i) las ideas novedosas y el conocimiento se encuentran distribuidos entre los agentes del sistema de innovación; (ii) incrementar la diversidad aumentaría la cantidad de alternativas; (iii) cuanto mayor sea la dimensión del contrato, mayores son las posibilidades de que el mismo sea otorgado a empresas que cuenten con un historial previo de cooperación con dicha agencia (Cabral et al., 2006).

Si además de estimular la innovación se quiere fomentar el emprendimiento, podemosdefinir un conjunto de alternativas. Por unlado, sepuedegarantizar que un porcentaje de las compras públicas esté reservado para las PYMEs o empresas de reciente creación (Karjalainen y Kemppainen, 2008), como ya ocurre en otros países como los Estados Unidos, Australia o los Países Bajos. La segunda de las opciones consiste en lo que aquí denominaremos como "separación coordinada" (ver Figura 1), con la cual se preserva el tamaño del mercado, ofreciendo de forma separada diversas licitaciones para las diversas tecnologías que se requieran en el producto. De esta forma, se podría incrementar la tasa de participación de PYMEs y start-ups (incluso ideas de proyecto provenientes de las universidades, centros de investigación, estudiantes, etc.), al mismo tiempo que se mantiene el incentivo del tamaño de mercado para que las grandes empresas también se vean incluidas.

Figura 1. La estrategia de la "separación coordinada"

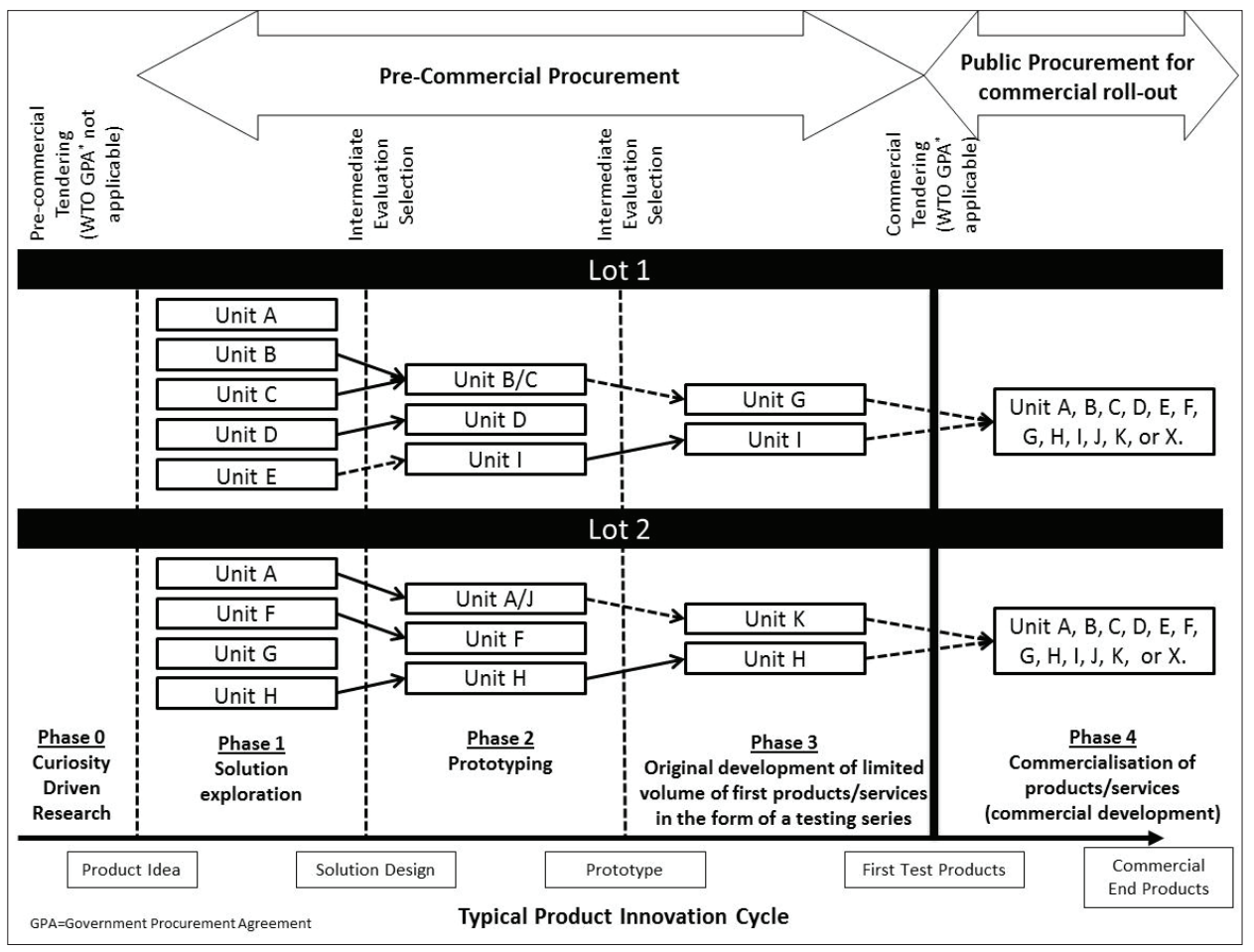

Fuente: Timmermans y Zabala-Iturriagagoitia (2013) 
Esta "separación coordinada" consta de dos dimensiones. La primera de ellas es la que denominamos como "separación horizontal". Acorde a ella, la convocatoria incluiría múltiples sub-convocatorias (de productos, de tecnologías, etc.), de modo que una empresa puede participar en una de dichas sub-convocatorias, en varias, o en todas. La segunda es la que denominamos como "separación vertical", ya que con ella se divide el proceso de innovación en varias etapas sucesivas. Así, un grupo de investigación podría participar en la etapa de diseño de la propuesta, pero sin embargo, no tendría por qué estar interesado en su fabricación, la cual podría ser desarrollada por una empresa manufacturera. Por supuesto, una misma empresa podría también presentarse a ambas convocatorias, tanto a la de diseño como a la de construcción.

El hecho de separar las etapas de exploración y de explotación es bien conocido (March, 1991; Greve, 2007). Ello responde a que no todas las empresas tienen recursos suficientes para participar en los dos procesos de forma simultánea. Hay que considerar asimismo que el conjunto de agentes que pueden participar en la generación de ideas es mayor que aquel centrado en la implementación y difusión de los productos, debido a las barreras de entrada existentes. Por tanto, el hecho de que la compra pública limite su ámbito de actuación al sector empresarial, nos parece que constituye una oportunidad aún sin explotar, ya que existe un claro potencial para relacionar agentes con competencias complementarias.

\section{Conclusiones y discusión}

En este artículo hemos definido lo que consideramos por CPI, mostrando una clasificación de los diferentes tipos de CPI que hemos ejemplarizado por medio de cuatro casos. Además de ello, también hemos realizado una propuesta de corte conceptual y metodológica por medio de la cual la compra pública puede actuar no sólo como estímulo a la innovación, sino también al emprendimiento (CPE). Con esta contribución sobre la CPE, nuestra intención es la de proporcionar un marco conceptual y metodológico para un campo de investigación que ha permanecido inexplorado. Por lo tanto, se requiere más investigación para profundizar en las implicaciones organizativas que la estrategia de separación coordinada puede tener para los organismos públicos encargados de los procesos de compra pública y las políticas de industriales y de innovación (Magro et al., 2014).

Otro área que requiere de mayor investigación futura es el relacionado con la evaluación de las políticas de innovación desde el lado de la demanda, un área que aún se encuentra en su infancia (Edler et al., 2012; Magro y Wilson, 2013). Sólo así será posible lograr una coevolución entre el desarrollo de la comunidad política de innovación y la práctica de su correspondiente evaluación.

Crecientemente, las políticas de articulación de la demanda se han ido complementando con otro tipo de instrumentos como la financiación de proyectos de $\mathrm{I}+\mathrm{D}$, incentivos fiscales, prospectiva tecnológica, regulaciones y estándares, etc. (Edler, 2009). Esto está directamente relacionado con el carácter multi-nivel y multi-agente de este tipo de políticas de innovación multi-instrumentales, a las cuales hay que concebir por tanto dentro de un 'policy-mix' más amplio que abarque varias iniciativas de carácter sistémico (Flanagan et al., 2011).

\section{Bibliografía}

Aschhoff, B. \& Sofka, W. (2009). Innovation on demand - Can public procurement drive market success of innovations?. Research Policy, 38(8), 1235-1247.

Berman, T. \& Squire, M. (2011). Demand-side innovation policies in Australia. En OECD (Ed.), Demand-side Innovation Policies (pp. 95113). Paris: OECD Publishing.

Bylund, J.R. (2006). Planning, Projects, Practice. A Human Geography of the Stockholm Local Investment Programme in Hammarby Sjöstad. $\mathrm{PhD}$ thesis, Department of Human Geography, Stockholm University.

Cabral, L., Cozzi, G., Denicoló, V., Spagnolo, G. \& Zanza, M. (2006). Procuring Innovations. En N. Dimitri, G. Piga \& G. Spagnolo (Eds.), Handbook of Procurement (pp. 483-528). Cambrige: Cambridge University Press.

Dalpé, R., DeBresson, C. \& Xiaoping, H. (1992). The public sector as first user of innovations. Research Policy, 21, 251-263.

Directorate for Emergency Communication (2007). Nødnett. New Public Safety Radio Network in Norway. Oslo.

Edler, J. (2009). Demand Policies for Innovation in EU CEE Countries. Manchester Business School Working Paper No 579, The University of Manchester.

Edler, J. \& Georghiou, L. (2007). Public Procurement and Innovation - Resurrecting the Demand Side. Research Policy, 36, 949-963.

Edler, J., Georghiou, L., Blind, K. \& Uyarra, E. (2012). Evaluating the demand side: New challenges for evaluation. Research Evaluation, 21(1), 33-47.

Edler, J., Georghiou, L., Uyarra, E. \& Yeow, J. (2015). The meaning and limitations of public procurement for innovation: a supplier's experience. En C. Edquist, N.S. Vonortas, J.M. Zabala-Iturriagagoitia \& J. Edler (Eds.), Public Procurement for Innovation (pp. 35-64). Cheltenham: Edward Elgar.

Edquist, C. (1997). Systems of innovation - Technologies, Institutions and Organizations. London: Pinter Publishers.

Edquist, C. (2005). Systems of Innovation: Perspectives and Challenges. En J. Fagerberg, D.C. Mowery \& R.R. Nelson (Eds.), The Oxford Handbook of Innovation (pp. 181-208). Oxford: Oxford University Press.

Edquist, C., Hommen, L. \& Tsipouri, L. (2000). Public Technology Procurement and Innovation. Boston/Dordrecht/London: Kluwer Academic Publishers.

Edquist, C., Vonortas, N.S., Zabala-Iturriagagoitia, J.M. \& Edler, J. (2015). Public Procurement for Innovation. Cheltenham: Edward Elgar. Edquist, C. \& Zabala-Iturriagagoitia, J.M. (2012). Public Procurement for Innovation as Mission-oriented Innovation Policy. Research Policy, 41(10), 1757-1769. 
Edquist, C. y Zabala-Iturriagagoitia, J.M. (2015). Pre-commercial procurement: a demand or supply policy instrument in relation to innovation?. RઐD Management, 45(2), 147-160.

European Commission (2006). Pre-commercial Procurement. A missing link in the European innovation cycle. European Commission, March 2006.

Flanagan, K., Uyarra, E. \& Laranja, M. (2011). Reconceptualising the 'policy mix' for innovation. Research Policy, 40(5), 702-713.

Geroski, P.A. (1990). Innovation, Technological Opportunity, and Market Structure. Oxford Economic Papers, 42, 586-602.

Gregersen, B. (1992). The Public Sector as a Pacer in National Systems of Innovation. En B.Å. Lundvall (Ed.), National Systems of Innovation - Towards a Theory of Innovation and Interactive Learning (pp. 129-145). London: Pinter.

Greve, H.R. (2007). Exploration and Exploitation in Product Innovation. Industrial and Corporate Change, 16(5), 945-975.

Gustafsson, R. \& Autio, E. (2011). A failure trichotomy in knowledge exploration and exploitation. Research Policy, 40, 819-831.

Hommen, L. (2005). Policy Recommendations for Innovationfriendly Public Technology Procurement. Deliverable 10, INNOUTILITIES Consortium.

Hommen, L. \& Rolfstam, M. (2009). Public Procurement and Innovation: towards a taxonomy. Journal of Public Procurement, 9, 17-56.

Kalvet, T. \& Lember, V. (2010). Risk management in public procurement for innovation: the case of Nordic-Baltic Sea cities. Innovation - The European Journal of Social Science Research, 23(3), 241-262.

Karjalainen, K. \& Kemppainen, K. (2008). The involvement of smalland medium-sized enterprises in public procurement: Impact or resource perceptions, electronic systems and enterprise size. Journal of Purchasing \& Supply Management, 14, 230-240.

Knafl, K.A. \& Howard, M.J. (1984). Interpreting and reporting qualitative research. Research in Nursing and Health, 7, 17-24.

Lember, V., Kalvet, T., Kattel, R., Penna, C. \& Suurna, M. (2007). Public Procurement for Innovation in Baltic Metropolises. Research report to BaltMet Inno Organization, Tallin.
Lember, V., Kalvet, T. \& Kattel, R. (2011). Urban Competitiveness and Public Procurement for Innovation. Urban Studies, 48(7), 1373-1395. Lyngstøl, T.H. (2004). TETRA pilot project. Final Report. Justis og Politidepartementet, Norwegian Public Safety Radio Project, July 2004.

March, J.G. (1991). Exploration and Exploitation in Organizational Learning. Organization Science, 2, 71-87.

Magro, E. \& Wilson, J.R. (2013). Complex Innovation Policy Systems: Towards an Evaluation Mix. Research Policy, 42(9),1647-1656.

Magro, E., Navarro, M. \& Zabala-Iturriagagoitia, J.M. (2014). Coordination-Mix: The Hidden Face of STI Policy. Review of Policy Research, 31(5), 367-389.

McCallie, D., Butts, J. \& Mills, R. (2011). Security analysis of the ADS$\mathrm{B}$ implementation in the next generation air transportation system. International Journal of Critical Infrastructure Protection, 4(2), 78-87.

OECD (2011). Demand side innovation policies. Paris: OECD Publishing.

Rothwell, R. \& Zegveld, W. (1981). Government regulations and innovation-industrial Innovation and Public Policy. En R. Rothwell \& W. Zegveld (Eds.), Industrial Innovation and Public Policy: Preparing for the 1980s and the 1990s (pp. 116-147). London: Pinter Publishers.

Shane, S.A. (2003). A General Theory of Entrepreneurship: The Individual-Opportunity Nexus. Cheltenham: Edward Elgar Publishing.

Sylvest, J. (2008). Opportunities for Public Technology Procurement in the ICT-related sectors in Europe. Final Report, Rambøll Management A/S, Commissioned by the European Commission, Directorate-General for Information Society and Media.

Timmermans, B. \& Zabala-Iturriagagoitia, J.M. (2013). Coordinated unbundling: A way to stimulate entrepreneurship through public procurement for innovation. Science and Public Policy, 40, 674-685.

Uyarra, E. \& Flanagan, K. (2009). Understanding the innovation impacts of public procuremen. European Planning Studies, 18, 123-143.

Vonortas, N.S. (2015). Innovation and public procurement in the United States. En C. Edquist, N.S. Vonortas, J.M. Zabala-Iturriagagoitia \& J. Edler (Eds.), Public Procurement for Innovation (pp. 147-178), Cheltenham: Edward Elgar.

Wintjes, R. (2012). Regional Innovation Monitor Thematic Paper 3, Demand-side innovation policies at regional level. Fraunhofer ISI and Technopolis Group, February 2012. 